\title{
Los datos abiertos como herramienta de aproximación a los parámetros de la morfología urbana. Caso de estudio: densidades urbanas en la Comunidad de Madrid
}

\author{
Javier Barros Guerton ${ }^{1}$, José María Ezquiaga Dominguez ${ }^{2}$ \\ Departamento de Urbanística y Ordenación del Territorio. \\ Universidad Politécnica de Madrid. Madrid, España \\ E-mail: 1javierbarrosguerton@live.com, 2jmezquiagadominguez@gmail.com,
}

\begin{abstract}
Resumen. La densidad es un parámetro central en el análisis y la regulación de los procesos urbanos; no obstante, su análisis se ha visto históricamente dificultado por la escasez de datos detallados y actualizados. La planificación urbanistica ha trabajado habitualmente sin contar con datos precisos sobre densidades edificadas, y con datos con niveles bastante altos de agregación en demografia y, sobre todo, empleo; el uso de nuevas fuentes de datos abiertos permite superar esta situación.

El Catastro estatal español aporta una fuente valiosa para el estudio de la intensidad edificatoria como soporte fisico de la densidad social, que a su vez se beneficia de una mayor frecuencia y detalle en la publicación de sus datos. En un trabajo sobre los datos abiertos del conjunto de la Comunidad de Madrid, una región con 6,5 millones de habitantes, se propone el análisis a través de SIG de las interrelaciones entre cuatro parámetros de densidad: edificatoria, de vivienda, poblacional y de ocupados en actividades económicas.

La investigación se inscribe en una secuencia centrada en la ciudad de Madrid, para la que el presente artículo aportará una contextualización territorial en la que los límites administrativos pierden importancia para facilitar la identificación de dinámicas de alcance metropolitano. La metodología empleada permitirá, en un futuro próximo, identificar tipologías de espacios urbanos que tienen potencial operativo de cara a la planificación.
\end{abstract}

Palabras clave: Densidad edificada, Morfología, Compacidad, Regiones Urbanas, Madrid, España

\section{Introducción}

El presente texto se organiza como una aproximación por escalas sucesivas a una pregunta: ¿Cómo puede describirse la densidad urbana en la Comunidad de Madrid?. Esta pregunta se enmarca en una ruta de investigación centrada en el municipio de Madrid, para la que los resultados suponen una aportación que permite contextualizar los valores.

La primera respuesta a esta pregunta se plantea en una comparación con tres territorios igualmente marcados por áreas metropolitanas. La segunda respuesta, en una escala más próxima, resulta de comparar Madrid con otras provincias españolas en situaciones contrastadas. Y la tercera es una visión interna a la propia región de Madrid, con un mayor detalle en cuanto a los aspectos morfológicos, e introduciendo además la dimensión temporal. La metodología empleada es una serie de comparaciones paramétricas entre diferentes escalas. Para ello se tiene en cuenta que por lo general los datos sobre densidad suelen reflejarse en función de unidades administrativas, debido 
a que muchos de los fenómenos, como los demográficos o los económicos, se miden a ese nivel; sin embargo, esa aproximación dificulta en gran medida la comparación cuando se trata de hablar de un parámetro como la intensidad edificatoria, pues a una misma forma urbana pueden corresponder densidades muy diferentes si, por ejemplo, se segrega una parte del municipio o se anexiona otra. En un análisis dentro de la línea "ciudad compacta versus ciudad difusa" esto cobra relevancia, pues los perímetros de análisis administrativos pueden ser muy variables. Por ello gran parte de los cálculos expresados se han realizado tomando como referencia, a partir de datos catastrales o asimilables, la huella edificada, y realizando una "operación acordeón", que puede verse en la imagen 1. Esta consiste en crear una equidistancia o buffer de 50 metros hacia el exterior de todas las huellas edificadas; agrupar esos perímetros en uno sólo; y volver a realizar una equidistancia del mismo valor, pero hacia el interior. Esta aproximación parte de la base de que, aunque las huellas edificadas reflejadas tienen presunción de precisión, pueden omitir en algunos casos construcciones auxiliares o partes subterráneas, y también pueden omitir áreas pavimentadas. Los polígonos generados en esa operación suponen una envolvente o huella, que puede resultar una aproximación a una superficie sellada más fácilmente comparable entre contextos diferenciados.

\section{Análisis}

La primera comparación pone a la misma escala a la región de Madrid con la ciudad de Nueva York (Estados Unidos), la región Île-deFrance (Francia) y el Distrito Capital de Bogotá (Colombia). La elección de estos casos se basa en la disponibilidad de datos abiertos que permitan abordar la cuestión, y el haber visitado en persona ese espacio. En todo caso, los datos disponibles tienen fuentes y metodologías diversas, pero también se refieren a espacios que no son funcionalmente equivalentes. En el caso de la Comunidad de Madrid y de Ilede-France el espacio analizado cubre el área metropolitana y espacios exteriores, pues son delimitaciones políticas de gran escala; en el caso de Nueva York la ciudad es sólo el centro de una gran área metropolitana que abarca tres estados, y en el de Bogotá, la extensión hacia el sur del Distrito Capital no compensa la intensa relación funcional con Cundinamarca hacia el norte y el oeste. En el caso de Nueva York los datos exteriores a la ciudad y localizados para este texto tanto dentro del propio estado como en Nueva Jersey y Connecticut no han permitido un análisis de área más amplia, y en el caso de Bogotá, los modelos de datos del IDECA para el Distrito Capital y del IGAC para Cundinamarca presentan diferencias.

El análisis de los datos sobre superficies construidas y densidades comparando a

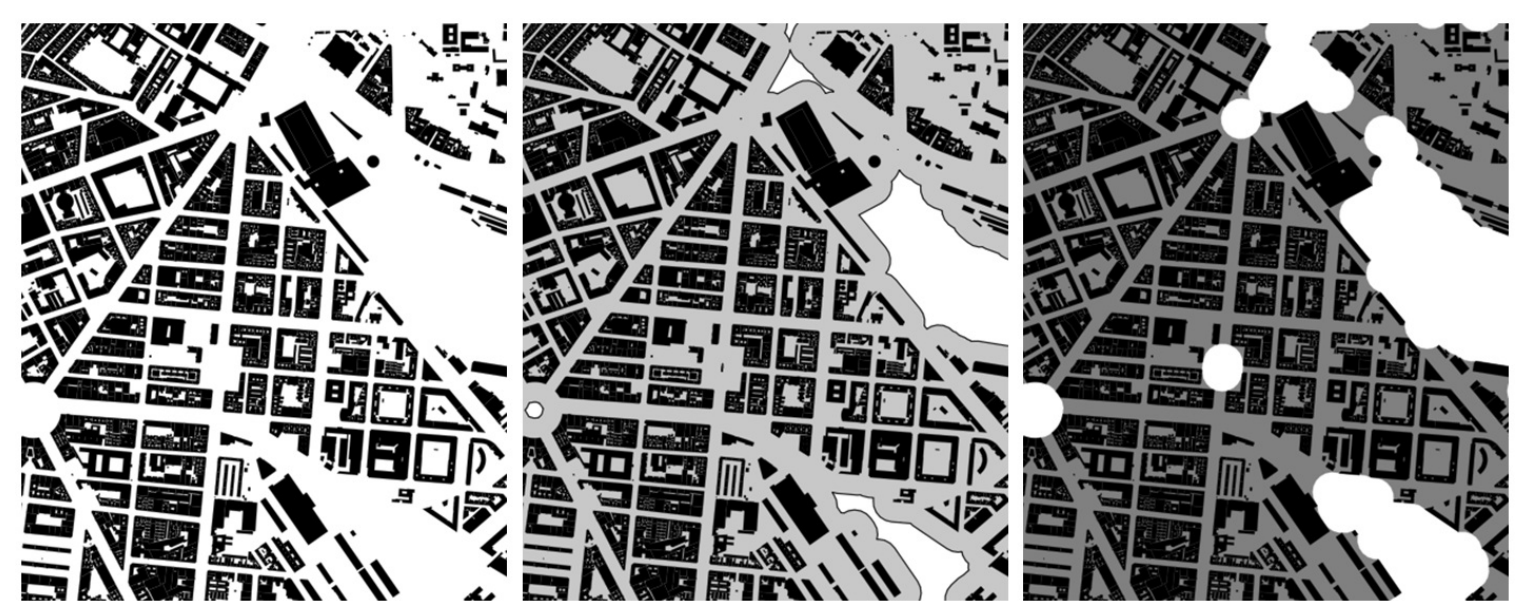

Fig. 1. Metodología de trazado de la huella de trabajo. De izquierda a derecha: ocupación en planta de los edificios según cartografía catastral, buffer de $50 \mathrm{~m}$ hacia el exterior, resultado final tras buffer de $50 \mathrm{~m}$ hacia el interior. Elaboración propia 
Madrid con los 3 ámbitos internacionales y las 3 provincias españolas escogidas muestra que, si se pondera con criterios morfológicos, la huella construida (que se aproxima a través de la huella descrita) tiene una densidad casi equivalente, en términos globales, en el conjunto de Madrid y en las áreas centrales de Nueva York o Bogotá; esto indica una elevada compacidad. Las provincias de Valencia y Barcelona presentan valores algo

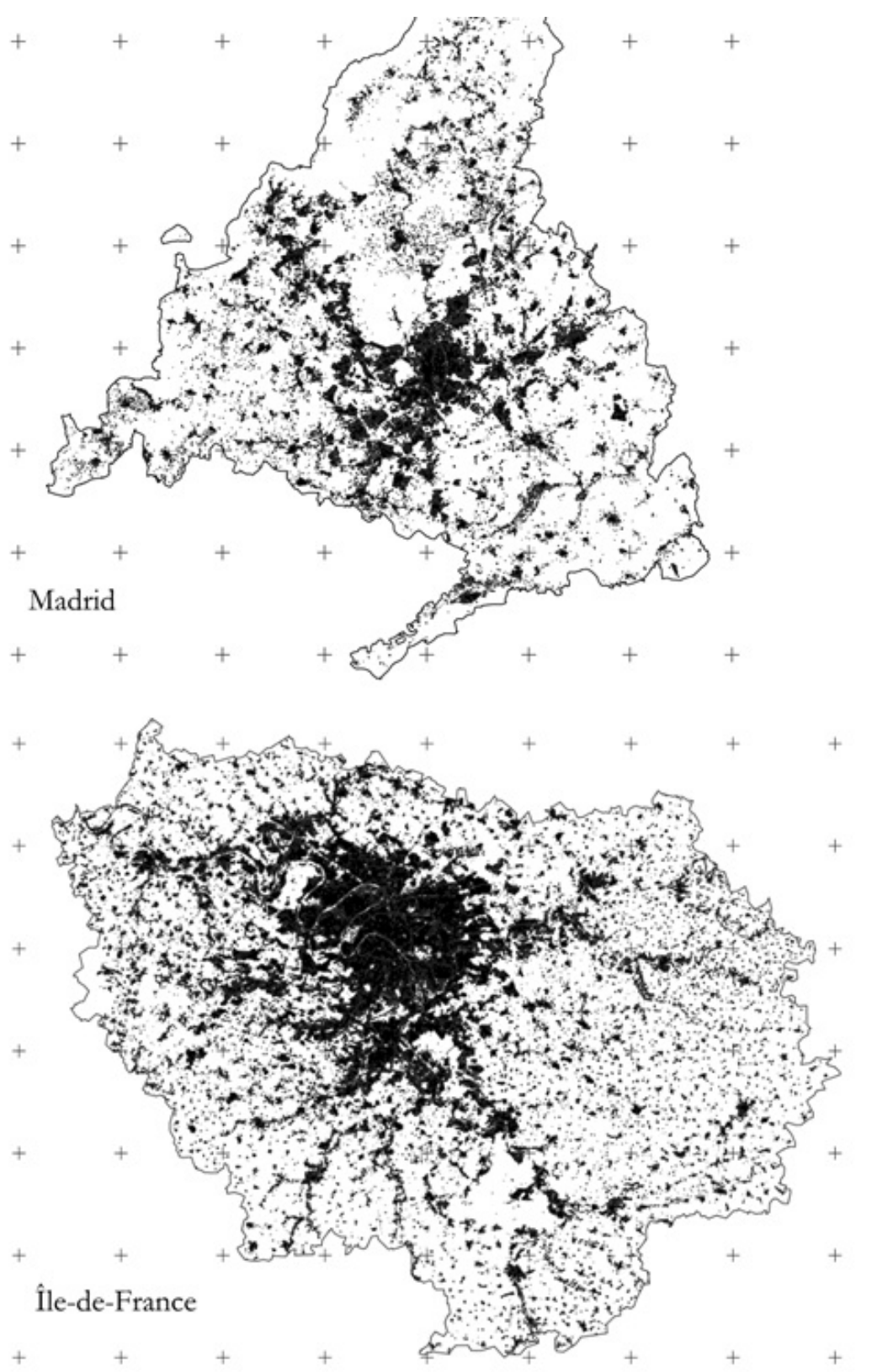

más reducidos, pero igualmente altos. En contraposición, la región Île-de-France y la provincia coruñesa presentan valores en torno a la mitad. En el primer caso, a la densidad del París central se contrapone una importantísima extensión periurbana unifamiliar de baja densidad, situación que también podría incidir significativamente en los casos colombiano y estadounidense de incluirse Nueva Jersey o Cundinamarca. En el caso corunés, la tradición

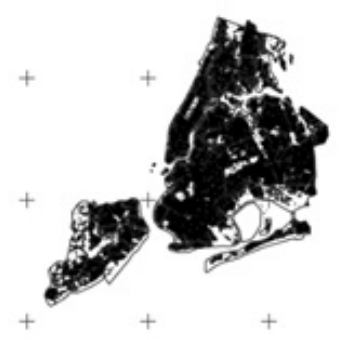

Nueva York

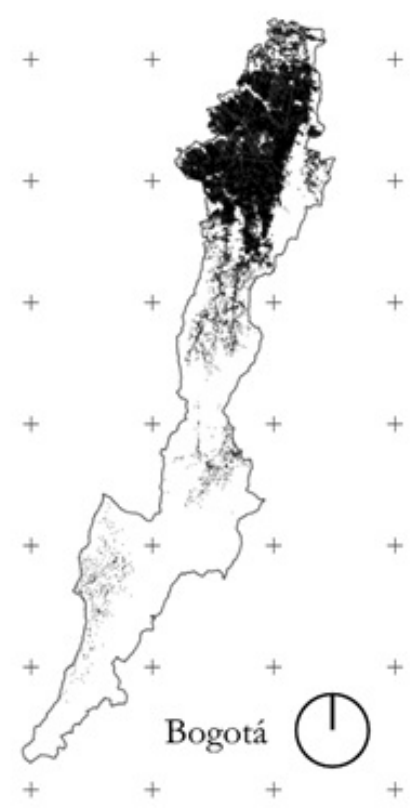

Fig. 2. Ámbitos de comparación internacional. Las marcas están espaciadas 20 km. Elaboración propia según datos abiertos de la Dirección General del Catastro español, el Department of City Planning de la Ciudad de Nueva York, el Cadastre du Ministere de l'Action et des Comptes Publics francés, y la Infraestructura de Datos Espaciales para el Distrito Capital de Bogotá. 


\begin{tabular}{|c|c|c|c|c|c|}
\hline & C total & C total $/$ hab & $\mathrm{C}$ vivienda $/ \mathrm{hab}$ & $\mathrm{C}$ total $/ \mathrm{m} 2$ político & $\mathrm{C}$ total/ $\mathrm{m} 2$ huella \\
\hline Île-de-France & 886.963 .528 & 73 & 44,40 & 0,0738 & 0,5150 \\
\hline Nueva York & 508.383 .979 & 61 & 38,32 & 0,6476 & 0,9970 \\
\hline $\begin{array}{l}\text { Bogotá } \\
\text { Comunidad de }\end{array}$ & 287.591 .817 & 35 & 16,29 & 0,1758 & 1,0213 \\
\hline Madrid & 625.073 .230 & 95 & 63,23 & 0,0778 & 0,9996 \\
\hline Barcelona (Provincia) & 606.431 .666 & 108 & 65,65 & 0,0702 & 0,8945 \\
\hline Valencia (Provincia) & 407.072 .990 & 160 & 93,75 & 0,0320 & 0,8488 \\
\hline Conuña (Provincia) & 183.146 .127 & 163 & 111,05 & 0,0230 & 0,5030 \\
\hline
\end{tabular}

Tab.1. Superficies construidas (suma de la de todas las plantas), expresadas como "C", totales y relativas a vivienda, y su relación con el volumen de población, con la extensión del ámbito político- administrativo, y con las superficies de huella calculadas. Elaboración propia según datos abiertos de la Dirección General del Catastro español, el Department of City Planning de la Ciudad de Nueva York, el Cadastre du Ministere de l'Action et des Comptes Publics francés y el Institut d'Urbanisme et d'Amenagement de la Région Île-de-France, y la Infraestructura de Datos Espaciales para el Distrito Capital de Bogotá.

de asentamiento disperso rural juega un papel importante en esa baja cifra, aún contando con la elevadísima densidad construida del municipio capital.

En lo relativo a la superficie construida total por habitante, resulta llamativo el bajo valor de Bogotá, probablemente vinculado a una renta per cápita más baja. Parece intuitivo que el valor medio de Île-de-France sea más elevado que el de Nueva York al incluir espacios periurbanos con precios del suelo y la construcción más bajos. Y resulta llamativo que los valores provinciales españoles resulten mucho más elevados; en los casos valenciano y corunés una cierta tradición de dispersión probablemente contribuye a estos valores, pero son más del doble del valor de París. El análisis de estos mismos datos sobre provincias de lo que en los últimos años se denomina España vacía, correspondiente a los territorios interiores que han experimentado un intenso proceso de despoblación durante el siglo XX, y en los que claramente no hay fenómenos metropolitanos, muestra que se pueden superar los $300 \mathrm{~m} 2$ construidos por habitante (324 en Teruel, 281 en Cuenca), pero que la densidad de la huella construida, que supone una aproximación a la compacidad, está en valores ligeramente superiores a los de la provincia coruñesa $(0,55$ y 0,64 , respectivamente).

Se aprecia en todo caso que el límite político- administrativo, si bien permite una aproximación interesante en cuanto a estimar el grado de ocupación del mismo y sobre la presión ejercida sobre el medio físico, no permite una lectura comparable sobre como se organiza la mancha urbana.

El análisis de las provincias españolas seleccionadas teniendo en cuenta los datos territorializados por municipios del Impuesto sobre la Renta de las Personas Físicas, que es indicativo de la economía de los residentes, pero no de las actividades, muestra que los municipios de cabecera de las áreas urbanas concentran una mayor renta disponible y que los municipios no adscritos a áreas urbanas tienen en promedio rentas disponibles menores. También se aprecia que a medida que aumenta la superficie construida por habitante tiende a disminuir la renta, tendencia que se verifica no sólo por las agregaciones señaladas sino también por municipios. Es decir, el mero hecho de incrementar el parque edificado de un municipio no implica, per se, mayor riqueza para los residentes, sino que esta depende de las actividades que realicen estos habitantes, dentro o fuera del municipio. Frente a los $184 €$ de renta disponible por $\mathrm{m} 2$ edificado (S(Renta disponible media $\times \mathrm{n}^{\mathrm{o}}$ declarantes)/ S(superficie construida total)) en el municipio de Barcelona, o los 176 en el de Madrid, la media en los municipios no incluidos en áreas urbanas de la provincia coruñesa es de $38 € /$ $\mathrm{m} 2$, valor apenas superados por los 41 de los municipios de pequeña población del área urbana de Gandía. Estos datos requieren, en todo caso, un contraste posterior, no abordado aquí, para tener en cuenta las rentas generadas por actividades económicas en cada municipio, y especialmente en el caso de municipios con 


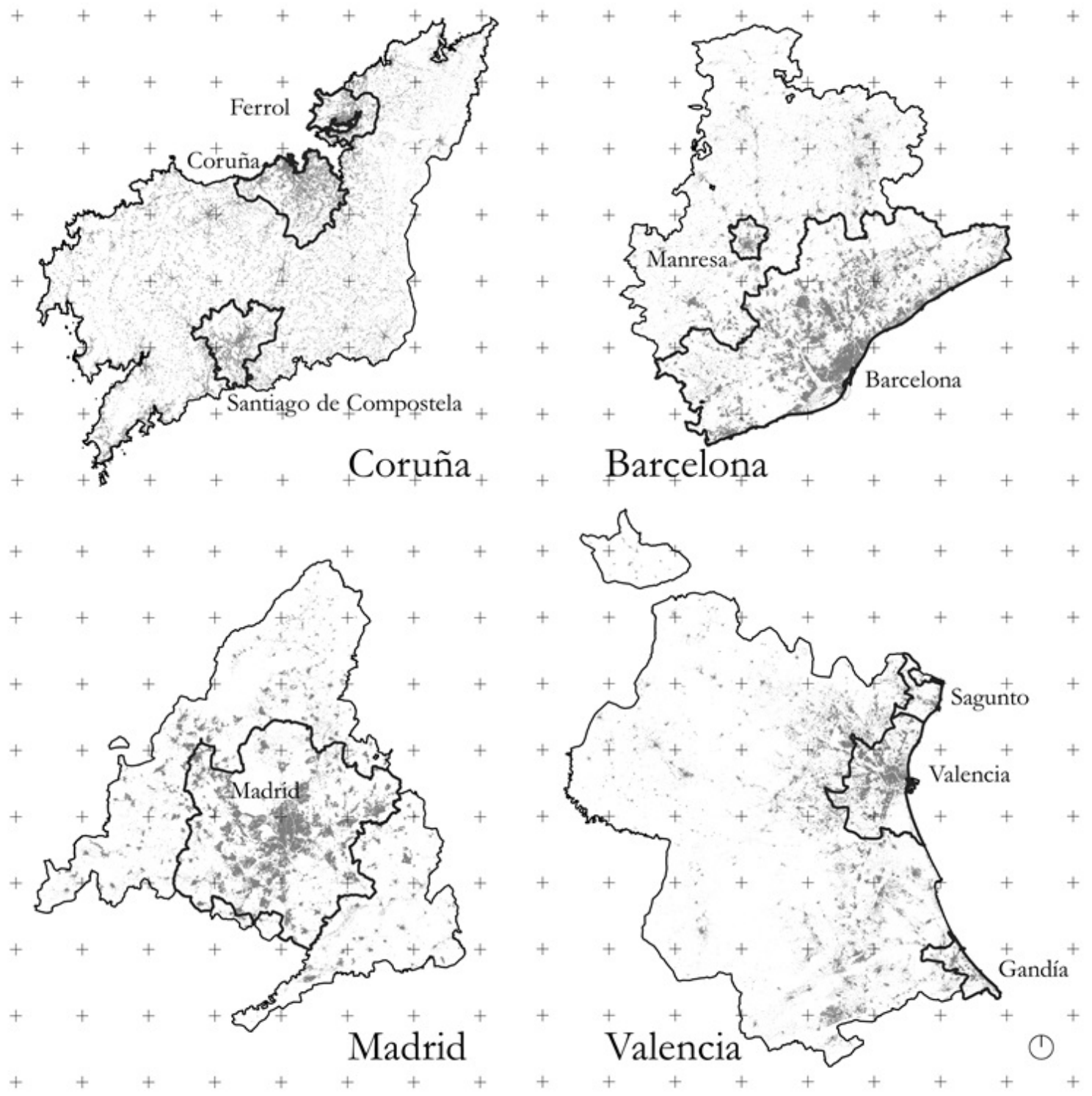

Fig. 3. Ámbitos de comparación nacional por provincias, con la delimitación de las Áreas Urbanas definidas por el Ministerio de Fomento. Las posiciones geográficas de las provincias han sido alteradas por facilidad de representación. Las marcas están espaciadas $20 \mathrm{~km}$. Elaboración propia según datos abiertos de la Dirección General del Catastro

fuerte presencia del turismo.

El análisis por provincias completas, atendiendo a la superficie construida, asignando a cada edificio su uso mayoritario según seis categorías (residencial, agrícola, industrial, oficinas, comercio y equipamientos), muestra que, pese a sus diferencias, los parques edificados de estas 4 provincias presentan características similares, con en torno a dos tercios de la superficie construida en edificios con uso mayoritario residencial; se aprecia claramente que el peso de los elementos agrícolas es mayor en Coruña, con más áreas rurales. El peso de las oficinas (siempre en edificio exclusivo) es claramente más relevante en Madrid, donde en el municipio de cabecera representan un 7\% de la superficie construida y en otros nueve se supera ese valor, mientras en Barcelona como provincia se alcanza un 3,2\%, con un 5,8\% en la capital y un valor superior en otros 12 municipios. En todo caso, el análisis a nivel de usos más desagregados y por debajo de la escala del edificio puede introducir matices importantes. 


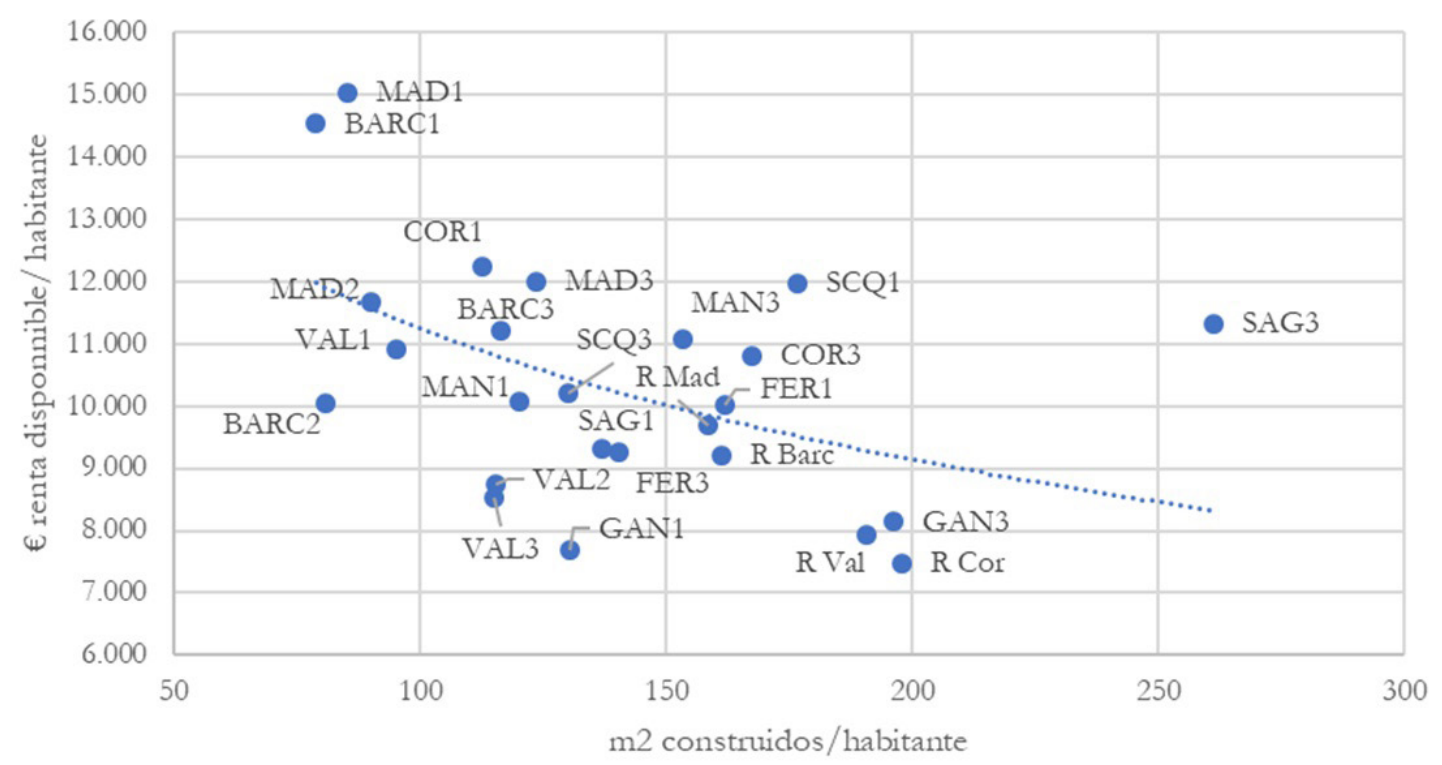

Fig. 4. Relación entre $\mathrm{m} 2$ construidos por habitante según el catastro de 2018 y renta disponible por habitante en $€$ según datos del IRPF de 2016, por ámbitos geográficos, y curva de regresión. BARC: Barcelona, MAN: Manresa, COR: Coruña, FER: Ferrol, SCQ: Santiago de Compostela, MAD: Madrid, GAN: Gandía, SAG: Sagunto, VAL: Valencia. El sufijo 1 indica municipio central del área urbana, 2 municipio del área urbana con más de 50.00 habitantes, 3 otros municipios del área urbana. El prefijo R indica otros municipios de la provincia sin adscripción a las áreas urbanas señaladas. Elaboración propia

El análisis más específico a nivel de la Comunidad de Madrid implica profundizar en las condiciones de la edificación desde el nivel de la sección censal, la unidad estadística de mayor nivel de detalle del sistema estadístico español. Dichas secciones se utilizan con fines electorales y censales, con un tamaño tal que abarca unas 1.000 personas por término medio; esto hace que su extensión territorial sea muy variable, por lo que no es una unidad que aporte una aproximación satisfactoria en términos de densidad. El Instituto Nacional de Estadística publica anualmente datos de población por secciones censales a través del padrón continuo , y el Instituto de Estadística de la Comunidad de Madrid publica, por el mismo ámbito, datos sobre ocupación y establecimientos. Por ello se plantea aplicar el sistema de análisis por huella a través de buffers en dos sentidos ya señalado, cruzado con las delimitaciones de secciones, entendiendo que las actividades están asociadas a las áreas edificadas; dada la baja participación del sector agropecuario en la economía y el empleo madrileños, esta hipótesis parece razonable.

Atendiendo a estas premisas y aprovechando que los datos catastrales permiten identificar la altura de las construcciones por cuerpos edificados, por debajo de la escala de la parcela, se plantea un análisis en el que los números máximos por sección censal ponderada de plantas sobre rasante y bajo rasante permiten caracterizar los espacios atendiendo a criterios morfológicos. Las siguientes tablas muestran la caracterización de las 4.345 secciones censales de Madrid atendiendo a esos dos parámetros, mostrando para cada combinación como se distribuyen la edificabilidad total y diferentes grupos de población.

Las cifras muestran que, por ejemplo, el $48 \%$ de la superficie construida en la Comunidad de Madrid lo está en secciones censales en las que hay al menos un edificio de baja más ocho o más alturas, en las que los coeficientes de edificabilidad están en general por encima de $1,1 \mathrm{~m} 2 / \mathrm{m} 2$, donde la edad media de la edificación es de cerca de 40 años; el $61 \%$ de los ocupados de la región trabajan en esos ámbitos, en los que residen el $46 \%$ de los habitantes, el $43 \%$ de los menores de 5 años y el $53 \%$ de los mayores de 65 . Si se introduce la variable de sótanos, en los 352 ámbitos con 


\begin{tabular}{|c|c|c|c|c|c|c|}
\hline & Vivienda & Agricultura & Industria & Oficinas & Comercio & Equipamientos \\
\hline $\begin{array}{l}\text { Comunidad de } \\
\text { Madrid }\end{array}$ & $66,9 \%$ & $2,5 \%$ & $9,5 \%$ & $5,2 \%$ & $4,0 \%$ & $11,9 \%$ \\
\hline Barcelona (Provincia) & $68,4 \%$ & $2,5 \%$ & $14,2 \%$ & $3,2 \%$ & $3,8 \%$ & $7,8 \%$ \\
\hline Valencia (Provincia) & $69,4 \%$ & $3,9 \%$ & $15,0 \%$ & $1,5 \%$ & $2,8 \%$ & $7,4 \%$ \\
\hline Coruña (Provincia) & $70,3 \%$ & $6,2 \%$ & $10,0 \%$ & $1,3 \%$ & $3,0 \%$ & $9,2 \%$ \\
\hline
\end{tabular}

Tab.2. Reparto de las superficies construidas en 2018 por uso predominante del edificio. Elaboración propia según datos abiertos de la Dirección General del Catastro.

\begin{tabular}{|c|c|c|c|c|c|}
\hline & & 1 sótano & 2 sótanos & 3 sótanos & Más de 3 sótanos \\
\hline Baja & & $0,00 \%$ & & & \\
\hline $\mathrm{Baja}+1$ & $0,00 \%$ & $0,00 \%$ & & & \\
\hline $\mathrm{Baja}+2$ & $0,01 \%$ & $0,92 \%$ & $0,04 \%$ & & \\
\hline $\mathrm{Baja}+3$ & $0,09 \%$ & $5,66 \%$ & $2,97 \%$ & $0,14 \%$ & \\
\hline $\mathrm{Baja}+4$ & $0,17 \%$ & $4,67 \%$ & $5,41 \%$ & $0,88 \%$ & $0,10 \%$ \\
\hline Baja+5 & $0,15 \%$ & $4,10 \%$ & $5,12 \%$ & $2,45 \%$ & $0,70 \%$ \\
\hline Baja+6 & $0,14 \%$ & $2,03 \%$ & $4,56 \%$ & $2,07 \%$ & $0,90 \%$ \\
\hline $\mathrm{Baja}+7$ & $0,08 \%$ & $1,46 \%$ & $2,64 \%$ & $2,54 \%$ & $1,58 \%$ \\
\hline Baja +8 & $0,11 \%$ & $1,08 \%$ & $3,19 \%$ & $2,98 \%$ & $1,64 \%$ \\
\hline Más de 9 alturas & $0,74 \%$ & $4,37 \%$ & $7,80 \%$ & $9,94 \%$ & $16,56 \%$ \\
\hline
\end{tabular}

Tabla 3. Porcentaje de la edificabilidad total en la comunidad de Madrid por grupos de secciones

\begin{tabular}{|c|c|c|c|c|}
\hline & 1 sótano & 2 sótanos & 3 sótanos & Más de 3 sótanos \\
\hline Baja & 0,71 & & & \\
\hline Baja+1 & 0,62 & & & \\
\hline $\mathrm{Baja}+2$ & 0,32 & 0,54 & & \\
\hline $\mathrm{Baja}+3$ & 0,37 & 0,37 & 0,39 & \\
\hline $\mathrm{Baja}+4$ & 0,47 & 0,62 & 0,64 & 0,85 \\
\hline $\mathrm{Baja}+5$ & 0,73 & 0,94 & 0,91 & 1,29 \\
\hline Baja+6 & 0,98 & 0,99 & 1,30 & 1,25 \\
\hline $\mathrm{Baja}+7$ & 0,93 & 1,27 & 1,32 & 1,58 \\
\hline Baja +8 & 1,20 & 1,24 & 1,57 & 1,96 \\
\hline Más de 9 alturas & 1,13 & 1,37 & 1,82 & 2,79 \\
\hline
\end{tabular}

Tabla 4. Coeficiente de edificabilidad global para cada grupo de secciones ponderadas

\begin{tabular}{|c|c|c|c|c|c|}
\hline & Sin sótano & 1 sótano & 2 sótanos & 3 sótanos & Más de 3 sótanos \\
\hline Baja & & 2011 & & & \\
\hline Baja+1 & 1997 & 1961 & & & \\
\hline $\mathrm{Baja}+2$ & 1944 & 1984 & 1985 & & \\
\hline $\mathrm{Baja}+3$ & 1975 & 1983 & 1988 & 1988 & \\
\hline $\mathrm{Baja}+4$ & 1967 & 1980 & 1986 & 1980 & 1978 \\
\hline$B a j a+5$ & 1968 & 1975 & 1983 & 1982 & 1986 \\
\hline Вајa+6 & 1970 & 1975 & 1983 & 1981 & 1967 \\
\hline $\mathrm{Baja}+7$ & 1970 & 1972 & 1977 & 1977 & 1964 \\
\hline Baja +8 & 1968 & 1970 & 1981 & 1974 & 1966 \\
\hline Más de 9 alturas & 1974 & 1973 & 1975 & 1975 & 1968 \\
\hline
\end{tabular}

Tabla 5. Edad promedio de la edificación en las secciones censales

\begin{tabular}{|c|c|c|c|c|c|}
\hline & Sin sótano & 1 sótano & 2 sótanos & 3 sótanos & Más de 3 sótanos \\
\hline \multicolumn{6}{|l|}{ Baja } \\
\hline Baja+1 & $0,00 \%$ & $0,00 \%$ & & & \\
\hline Baja+2 & $0,00 \%$ & $0,34 \%$ & $0,01 \%$ & & \\
\hline $\mathrm{Baja}+3$ & $0,04 \%$ & $2,46 \%$ & $1,60 \%$ & $0,15 \%$ & \\
\hline Baja+4 & $0,12 \%$ & $2,07 \%$ & $3,76 \%$ & $0,88 \%$ & $0,09 \%$ \\
\hline $\mathrm{Baja}+5$ & $0,05 \%$ & $2,65 \%$ & $3,29 \%$ & $2,56 \%$ & $1,12 \%$ \\
\hline Ваја+6 & $0,06 \%$ & $1,64 \%$ & $2,96 \%$ & $2,11 \%$ & $2,00 \%$ \\
\hline $\mathrm{Baja}+7$ & $0,04 \%$ & $1,25 \%$ & $2,13 \%$ & $2,60 \%$ & $2,95 \%$ \\
\hline Baja+8 & $0,09 \%$ & $0,83 \%$ & $3,06 \%$ & $2,56 \%$ & $3,11 \%$ \\
\hline Más de 9 alturas & $0,39 \%$ & $3,14 \%$ & $6,98 \%$ & $13,88 \%$ & $27,04 \%$ \\
\hline
\end{tabular}

Tabla 6. Porcentaje de ocupados por grupo de secciones

Fig. 3,4,5 y 6. Tabla 3. Porcentaje de la edificabilidad total en la comunidad de Madrid por grupos de secciones, Tabla 4. Coeficiente de edificabilidad global para cada grupo de secciones ponderadas, Tabla 5. Edad promedio de la edificación en las secciones censales, Tabla 6. Porcentaje de ocupados por grupo de secciones 


\begin{tabular}{|c|c|c|c|c|c|}
\hline & Sin sótano & 1 sótano & 2 sótanos & 3 sótanos & Más de 3 sótanos \\
\hline Baja & & $0,02 \%$ & & & \\
\hline Baja+1 & $0,05 \%$ & $0,01 \%$ & $0,00 \%$ & & \\
\hline Вaja+2 & $0,02 \%$ & $0,69 \%$ & $0,04 \%$ & & \\
\hline $\mathrm{Baja}+3$ & $0,25 \%$ & $4,22 \%$ & $2,32 \%$ & $0,11 \%$ & \\
\hline $\mathrm{Baja}+4$ & $0,44 \%$ & $5,10 \%$ & $4,71 \%$ & $0,77 \%$ & $0,12 \%$ \\
\hline $\mathrm{Baja}+5$ & $0,36 \%$ & $5,71 \%$ & $6,47 \%$ & $2,19 \%$ & $0,54 \%$ \\
\hline Ваја+6 & $0,35 \%$ & $2,97 \%$ & $5,13 \%$ & $2,34 \%$ & $0,56 \%$ \\
\hline $\mathrm{Baja}+7$ & $0,19 \%$ & $1,66 \%$ & $2,98 \%$ & $2,26 \%$ & $1,16 \%$ \\
\hline Baja +8 & $0,26 \%$ & $1,84 \%$ & $3,38 \%$ & $2,74 \%$ & $1,12 \%$ \\
\hline Más de 9 alturas & $1,63 \%$ & $7,29 \%$ & $9,90 \%$ & $10,47 \%$ & $7,65 \%$ \\
\hline \multicolumn{6}{|c|}{ Tabla 7. Porcentaje de habitantes por grupos de secciones } \\
\hline & Sin sótano & 1 sótano & 2 sótanos & 3 sótanos & Más de 3 sótanos \\
\hline Baja & & $0,06 \%$ & & & \\
\hline Baja+1 & $0,15 \%$ & $0,01 \%$ & $0,00 \%$ & & \\
\hline Baja+2 & $0,01 \%$ & $0,50 \%$ & $0,03 \%$ & & \\
\hline $\mathrm{Baja}+3$ & $0,21 \%$ & $4,03 \%$ & $2,41 \%$ & $0,09 \%$ & \\
\hline Baja+4 & $0,41 \%$ & $4,90 \%$ & $5,54 \%$ & $0,71 \%$ & $0,11 \%$ \\
\hline Baja+5 & $0,30 \%$ & $5,25 \%$ & $7,08 \%$ & $2,52 \%$ & $0,68 \%$ \\
\hline Baja+6 & $0,33 \%$ & $2,78 \%$ & $6,04 \%$ & $2,72 \%$ & $0,57 \%$ \\
\hline $\mathrm{Baja}+7$ & $0,18 \%$ & $1,61 \%$ & $3,47 \%$ & $3,27 \%$ & $1,11 \%$ \\
\hline Baja +8 & $0,21 \%$ & $1,51 \%$ & $3,70 \%$ & $3,45 \%$ & $1,14 \%$ \\
\hline Más de 9 alturas & $1,33 \%$ & $5,76 \%$ & $8,74 \%$ & $9,99 \%$ & $7,12 \%$ \\
\hline
\end{tabular}

Tabla 8. Porcentaje de menores de 5 años por grupos de secciones

\begin{tabular}{|c|c|c|c|c|c|}
\hline \multicolumn{2}{|c|}{ Sin sótano } & 1 sótano & 2 sótanos & \multirow[t]{2}{*}{3 sótanos } & Más de 3 sótanos \\
\hline Baja & & $0,00 \%$ & & & \\
\hline $\mathrm{Baja}+1$ & $0,00 \%$ & $0,01 \%$ & $0,00 \%$ & & \\
\hline Baja+2 & $0,03 \%$ & $0,64 \%$ & $0,04 \%$ & & \\
\hline $\mathrm{Baja}+3$ & $0,24 \%$ & $3,47 \%$ & $1,77 \%$ & $0,09 \%$ & \\
\hline Baja+4 & $0,56 \%$ & $4,62 \%$ & $3,52 \%$ & $0,78 \%$ & $0,13 \%$ \\
\hline $\mathrm{Baja}+5$ & $0,46 \%$ & $5,69 \%$ & $5,36 \%$ & $2,08 \%$ & $0,48 \%$ \\
\hline Baja+6 & $0,38 \%$ & $2,75 \%$ & $4,03 \%$ & $1,94 \%$ & $0,58 \%$ \\
\hline Baja +7 & $0,25 \%$ & $1,66 \%$ & $2,25 \%$ & $1,68 \%$ & $1,15 \%$ \\
\hline Baja +8 & $0,36 \%$ & $2,30 \%$ & $2,79 \%$ & $2,21 \%$ & $1,12 \%$ \\
\hline Más de 9 alturas & $2,15 \%$ & $9,49 \%$ & $11,23 \%$ & $12,52 \%$ & $9,18 \%$ \\
\hline
\end{tabular}

Tabla 9. Porcentaje de mayores de 65 años por grupos de secciones

Fig. 7,8 y 9. Tabla 3. Tabla 7. Porcentaje de habitantes por grupos de secciones, Tabla 8. Porcentaje de menores de 5 años por grupos de secciones, Tabla 9. Porcentaje de mayores de 65 años por grupos de secciones

al menos un edificio con más de 9 alturas y más de 3 sótanos está el $16,6 \%$ de la superficie edificada, la edificabilidad media supera 2,79 $\mathrm{m} 2 / \mathrm{m} 2$, la edad promedio de la edificación es de 50 años y trabaja el $27 \%$ de los ocupados; sólo vive el 7,65\% de los madrileños, el 7,12\% de los menores de 5 años y el $9,18 \%$ de los mayores de 65.

Por último, el sistema de información catastral incluye actualizaciones periódicas. Los datos publicados en 2014 a nivel global para el conjunto de las provincias gestionadas por el Ministerio de Hacienda mostraba para la Comunidad de Madrid un total de 580,8 millones de $\mathrm{m} 2$ construidos; los datos para 2018, según los trabajos realizados para este escrito, serían 624,8 millones, y la explotación de los datos publicados en abril de 2019 arroja un valor de 630,6 millones. El análisis en detalle de estas cifras muestra que se modifican tanto por demoliciones y nuevas construcciones como por correcciones de errores de transcripción, que pese al alto nivel de calidad de la base catastral están presentes en un número reducido.

\section{Conclusión}

La comparación internacional y nacional muestra que la Comunidad de Madrid tiene una huella relativamente compacta, aunque con importantes variaciones internas en su intensidad de edificación y de usos. Los datos abiertos utilizados han permitido realizar una caracterización inicial de la huella de la Comunidad de Madrid en su conjunto, y la metodología permite llevar dicho análisis a un nivel mucho más detallado, de sección censal. La continuidad de la investigación permitirá un ajuste de los parámetros para el apoyo a la 
toma de decisiones de ordenación.

El uso de fuentes de diversas procedencias ha mostrado la relevancia de tener en cuenta las condiciones específicas de producción de cada una, lo que hace que la comparación sólo pueda hacerse sobre parámetros muy acotados, como la superficie construida o las alturas. Son ciertamente imposibles, incluso dentro de España, cálculos precisos de la superficie urbanística computable, dado que aunque la metodología catastral se presenta como homogénea, en la práctica cada delegación introduce ciertos sesgos, y además su propósito es fiscal, por lo que mide sólo aquello que tributa; por otra parte, cada municipio tiene sus propios criterios para medir la superficie computable, lo que hace el trabajo sobre un grupo amplio particulamente complicado. Ello no resta interés al análisis, incluso a nivel urbano, pues la fuente catastral es la más pormenorizada; en el ámbito de la planificación territorial esta comparación transversal cobra mayor interés si cabe, pues en esa escala son, a menudo, más relevantes las relaciones $\mathrm{y}$ equilibrios entre variables más que los valores absolutos.

El cruce de los valores socieoconómicos permite una ponderación importante de los parámetros morfológicos físicos; las discordancias entre fechas de datos de uno y otro tipo se ven relativamente amortiguados por la mucho mayor inercia, al menos en el momento actual, del parque edificado respecto de los fenómenos socioeconómicos.

\section{Notas}

1 La Comunidad de Madrid es una comunidad autónoma que abarca una única provincia, y a efectos de comparación internacional tiene rango de región con capacidad legislativa, entre otras, en materia urbanística y de ordenación del territorio

2 http://www.catastro.meh.es/esp/acceso infocat.asp . Se han utilizado datos en formato inspire de 2018 (todas las zonas) y 2019 (Comunidad de Madrid)

3 https:/www1.nyc.gov/site/planning/datamaps/open-data.page\#zoning_related. Se ha utilizado mappluto en su versión 18-2-1

4 https://cadastre.data.gouv.fr/ , http:// data.iau-idf.fr/ . Los datos catastrales son de 2019, y los de edificabilidad, de los Îlots Morphologiques Urbains, datan de 2014.

5 https://www.ideca.gov.co/

6 https://www.agenciatributaria.es/AEAT. internet/datosabiertos/catalogo/hacienda/ Estadistica_de_los_declarantes_del_IRPF por_municipios.shtml

7 El padrón municipal de habitantes registra a los habitantes del municipio. El enlace informático entre municipios hace que, por ejemplo, los cambios de residencia de uno a otro se registren a través del alta en el nuevo, que implica la baja automática en el anterior. Asumiendo su diferencia metodológica con el censo decenal, resulta una aproximación operativa de mayor frecuencia a la cifra de población

8 Observatorio de Vivienda y Suelo. Boletín Especial Censo 2011 Parque edificatorio. Dirección General de Arquitectura, Vivienda y Suelo; Ministerio de Fomento. Abril 2014

\section{Referencias}

Mercadé Aloy, J., Magrinya Torner, F, Cervera Alonso de Medina, M. 2018. "Descifrando la forma urbana. Un análisis de patrones de agrupamiento basado en SIG.". GeoFocus (Artículos), no 22, P3-19. ISSN: 1578-5157. http:dx.doi.org/10.21138/GF.612

Fusco, Giovanni y Araldi, Alessandro. 2017. "The Nine Forms of the French Riviera: Classifying Urban Fabrics from the Pedestrian Perspective". En 24th ISUF International Conference. Book of Papers. Editorial Universitat Politècnica de València. 1313-1325. doi:10.4995/ ISUF2017.2017.5219

Cordeau, Erwan. 2016. Les îlots morphologiques urbains (IMU). Délimitation et caractérisation des «IMU 2012 » en Île-de-France. Institut d'Amenagement et d'Urbanisme d'Île-de-France. https://www. iau-idf.fr/nos-travaux/publications/les-ilotsmorphologiques-urbains-imu.html

AAVV. 2016. Future of Cities: The Science of Cities and Future Research Priorities. A report by the project's Lead Expert Group. Foresight, Government Office for Science Angel, S. 2015. The rise and fall of Manhattan's 
densities, 1800-2010. Marron Institute of Urban Management. NYU. https:// marroninstitute.nyu.edu/uploads/content/ Manhattan_Densities_High_Res, 1 January_2015.pdf

García Martín, Fernando Miguel. 2014. "Estudios de Forma Urbana en la Región de Murcia. Densidad y Compacidad del casco urbano de Lorca". In VII Anuario de jóvenes Investigadores. Universidad Politécnica de Cartagena

Dirección General de Arquitectura, Vivienda y Suelo .2014. "Boletín Especial Censo 2011 Parque edificatorio". Observatorio de Vivienda y Suelo. Ministerio de Fomento, Madrid, Abril 2014.

Batty, Michael. 2013. The New Science of Cities. The MIT Press.

AA VV .2013. "Policentrismo en los espacios urbanos. Aglomeraciones urbanas con varios centros/polos históricos. ¿Qué está pasando en España?”. Número Especial de Ciudad y Territorio. Vol XLV. Cuarta época. No 176. Verano 2013.

Ezquiaga Domíngez, José María, Barros Guerton, Javier, y Peribañez Ayala, Gemma. 2013. "La rehabilitación como sector de futuro en Madrid". Barómetro de Economía de la Ciudad de Madrid, $\mathrm{n}^{\mathrm{o}} 35$, 1er trimestre 2013. https://www. madrid.es/UnidadesDescentralizadas/ $\mathrm{U} \mathrm{D} \mathrm{CO}$ b s e r v E c o n o m i c o/ BarometroEconomia/2013/Ficheros/Enero/ rehabilitacion.pdf

Sevtsuk, A., y Mekonnen, M. 2012. "Urban Network Analysis". A new toolbox for ArcGIS. Revue Internationale de Géomatique. http://doi.org/10.3166/ rig.22.287-305

Gallo, M.T.; Garrido, R.. 2012. "Una aproximación a la estructura urbana policéntrica en la Comunidad de Madrid". ACE: Architecture, City and Environment, 6 (18), pp 69-100

Temes Cordovez, Rafael. 2008. "Las fuentes catastrales y la identificación de las transformaciones en los tejidos urbanos". CT: Catastro, $n^{\circ}$ 64, Madrid, pp 55-78. Doi: http://hdl.handle.net/10251/11114

De Sola Morales, Manuel. 1993. Las formas del crecimiento urbano. Barcelona: Edició
UPC (Col. D'Arquitectura)

Alexander, C, Ishikawa, S., y Silverstein, M. 1977. A Pattern Language. http://doi. org/10.2307/1574526 